\title{
Japanese Translation Quality Evaluation System Based on Deep Neural Network Algorithm
}

\author{
Rui Zhang \\ School of Foreign Studies, Lingnan Normal University, Zhanjiang 524048, China \\ E-mail: ruiruirui_2021@163.com
}

Received: July 17, 2021. Revised: December 10, 2021. Accepted: January 11, 2022. Published: January 13, 2022.

\begin{abstract}
The current translation quality evaluation system relies on the combination of manual and text comparison for evaluation, which has the defects of low efficiency and large evaluation errors. In order to optimize the defects of the current quality evaluation system, a Japanese translation quality evaluation system based on deep neural network algorithm will be designed. In order to improve the processing efficiency of the system, the USB3.0 communication module of the hardware system will be optimized. Based on the hardware design, the reference translation map is used to extend the reference translation of Japanese translation. The evaluation indexes of over- and under-translation are set, and the evaluation of Japanese translation quality is realized after the parameters are determined by training the deep neural network using the sample set. The system functional test results show that the average data transmission processing time of the system is improved by about $31.27 \%$, and the evaluation error interval is smaller and the evaluation is more reliable.
\end{abstract}

Keywords-deep neural network, Japanese translation, translation quality, quality evaluation, system design, reference translation map.

\section{INTRODUCTION}

$\mathrm{I}^{\mathrm{N}}$ $\mathrm{N}$ the present era of big data and the environment of massive influx of information on the Internet, the research and development in the field of natural language processing has given birth to machine translation system, which can use computer to carry out efficient and large volume of automatic translation, and this The technology is rapidly gaining popularity. Translation quality evaluation helps to improve the performance of machine translation system, therefore, the rapid development of machine translation technology drives the continuous development of evaluation technology, at the same time, more and more evaluation activities also drive the translation evaluation technology forward, and the competitive evaluation activities promote the improvement of machine translation performance to a certain extent [1].

Manual evaluation is the most primitive means of evaluating the quality of machine translation, by manually checking the translations one by one to judge whether they are accurate or not. Although the results of manual evaluation are more accurate, this method also has many shortcomings: it is time-consuming, expensive and the results are not reproducible, and the evaluation results are more subjective. Some scholars mainly realize the evaluation by establishing evaluation rules and evaluation systems, mostly from a qualitative perspective. The above-mentioned evaluation methods are limited by the evaluation rules and the selection of indicators, and there are biases without evaluation, and they cannot provide specific parameters for quantitative improvement of machine translation [2]-[4]. Benkova et al. [5] compared the phrase-based and automatic metric-based and neural network-based translation quality evaluation methods. Translation quality in English and Slovak was assessed using automatic indicators. Residuals were used to compare scores of statistical machine translation to the precision of neural machine translation. Based on all neural machine translation, BLEU_n scores found better translation of news text from English to Slovak. The quality of Arabic translation was evaluated by Ali [6] using textual peer-to-peer model. English translation output to Arabic text was compared using three machine interpreters, Microsoft Bing, and Ginger. For the evaluation of the machine translation (MT) output, an English text and its Arabic counterpart were selected from the United Nations records. The English source text is split into 84 semantic blocks. Depending on the corresponding model text in Arabic, each block is rated as fidelity or incorrect on two levels of the translation properties ".For a quantitative description of the evaluation process, the number of fidelity and clarity errors and their percentages were calculated. Although this method has a high evaluation efficiency, the model requires in-depth and thorough post-editing, and has limitations when applied to different language translations. The results of foreign scholars, such as Stoykova and Majchrakova [7], Ganesh et al. [8], evaluated the quality of machine translation in Bulgarian. It uses information 
retrieval-inspired search techniques and uses parallel statistical search for server algorithm steps with a query extension in the Slovak-Bulgarian European corpus using the sketch engine software and its score. Search included using statistical scores to generate study keyword consistency, combination, word sketch differences, word sketches and thesiopoeia, and statistical scores were treated as intermediate (interverbal) semantic standard representations of the study keyword (from the source language) and its possible translation equivalents (mapped to the target language. However, the above methods still evaluate with a single index, which is difficult to evaluate translation quality comprehensively [9]-[11].

In order to improve the evaluation effect of the translation quality and reduce the evaluation error, this paper proposes an evaluation system of the Japanese translation quality based on the deep neural network algorithm. Optimization of the hardware system, USB3.0 communication module was designed. The reference translation of the Japanese translation was extended using the reference translation diagram. After the evaluation indicators for overtranslation and undertranslation were set and the algorithm parameters were determined using the sample set, the evaluation of Japanese translation quality was achieved using deep neural networks.

Deep neural network, a branch of machine learning, is an algorithm that uses artificial neural networks as an architecture for learning representations of data [12]. Deep neural network algorithms are widely used in various fields, and evaluation using deep neural network algorithms can effectively improve processing efficiency. Therefore, the innovation point of this paper is the optimization design of the system software part using the deep neural network algorithm. The experimental results show that the evaluation quality of Japanese translation is improved, to promote the improvement of the translation ability and translation quality of machine translation.

\section{HARDWARE DESIGN OF JAPANESE TRANSLATION \\ QuALITY EVALUATION SYSTEM BASED ON DEEP NEURAL NETWORK ALGORITHM}

\section{A. Overall system hardware scheme}

The Japanese translation quality evaluation system designed in this paper needs to be able to compare and analyze a large number of translation references, so the hardware part of the system is required to support the high-speed data playback function. The hardware architecture of the system is shown in Figure 1.

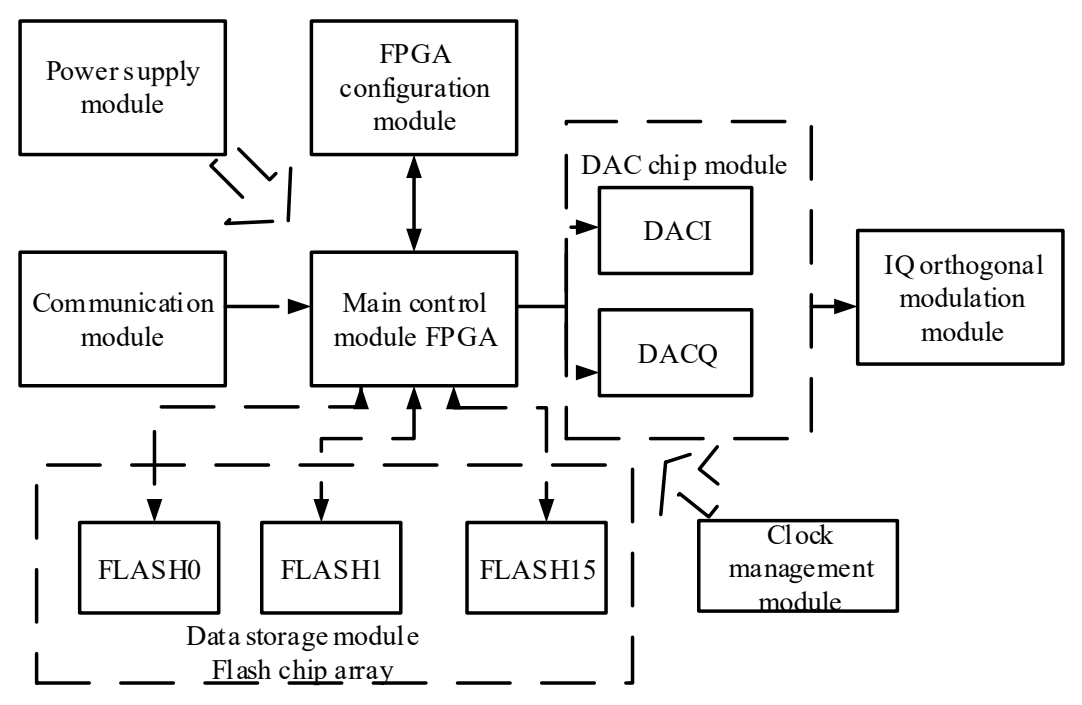

Figure 1. Hardware architecture block diagram of quality assessment system

The system mainly includes the main control module, DAC chip module, IQ quadrature modulation module, communication module, data storage module, clock management module, and power supply module. The following section will explain the chip selection of the main modules.

1. Main control module: The system's main control module uses FPGA chip EP3C10E144C8N, which is responsible for controlling peripheral modules such as USB3.0, DAC, FLASH, etc. The chip has 94 IOs, 10k logic gates and remote upgrade configuration mode for online reconfiguration function, which can meet the system's requirements for multi-port number, large cache capacity and high port performance. It can meet the system requirements for multiple ports, large cache capacity, and high-end port performance.

2. DAC chip module: the DAC chip module serves to convert digital signals into analog signals and consists of two DAC chips, each of which generates a differential analog signal as an I-way and Q-way signal. The 16-bit DAC5681z is selected, which has a sampling rate of up to 1 Gsps and provides true differential input, which provides better noise rejection, distortion improvement, and wider dynamic range than single-ended input [13].

3. IQ quadrature modulation module: IQ quadrature modulation module includes IQ modulation chip and band-pass filter. Its role is to modulate the I-way and Q-way signals from the DAC output for IQ modulation to 
the RF, and then the RF signal passes through the band-pass filter, and the filtered signal is used as the final signal of the system output. The system uses the IQ modulation chip TRF3702, a low-noise quadrature modulator, the input signal of the chip is two differential voltage signals or single-ended signals, the common mode voltage is $3.7 \mathrm{~V}$, the signal bandwidth is $700 \mathrm{MHz}$, the intrinsic input is a single-ended signal from $1.5 \mathrm{G}$ to $2.5 \mathrm{GHz}$. the DAC $5681 \mathrm{z}$ and TRF3702 are connected by a resistor network The differential current from the DAC output is converted to a differential voltage signal with a common mode voltage of 3.7 $\mathrm{V}$ through the resistor network [14].

4. Communication module: communication module is mainly responsible for the communication with the host computer, the system uses a high-speed, universal and extensive USB3.0 interface, and the host computer can transfer massive amounts of data through the USB3.0 interface to the hardware part of the system. Parsing the USB3.0 protocol uses the USB3.0 controller chip CYUSB3014, which is compatible with USB2.0 and has a wide range of applicability.

5. Data storage module: the data storage module is mainly used to store the data generated in the translation process and important data such as translation dictionary, which requires that the data will not be lost after the system power failure. The FLASH model is MT29F8G16, and the capacity of each FLASH chip is $8 \mathrm{~GB}$, considering the system requirement of $128 \mathrm{G}$ single board capacity, so the FLASH chip array consists of 16 FLASH chips [15].

6. Clock management module: The clock management module is responsible for providing clocks for each module of the system. the core clock of the USB chip is provided by a passive crystal, the system clock of the FPGA is provided by a TCXO temperature compensated crystal oscillator, the refresh clock of the DAC is generated by a voltage controlled crystal oscillator, and the clock of the accompanying path is provided by the FPGA, in order to ensure the synchronization of the two phases, the CDCM7005 chip is used In order to ensure the synchronization of the two phases, the CDCM7005 chip is used as the clock management chip. The clock generated by the voltage controlled crystal oscillator generates a multiplexed synchronous clock through this chip, which is then provided to the two DACs for use.

\section{B. Communication module design}

Since the system requires a large amount of information to be collected and processed in real time when evaluating the translation quality, this puts a lot of pressure on the system's data communication module. In order to improve the data processing transmission rate, this paper will develop and design the firmware for the CYUSB3014 of the communication module.

The CYUSB3014 initiates boot image file loading in several ways, and the boot method is selected by configuring the PMODE pins. The boot method settings are shown in Table 1 [16].

Table 1. Boot method settings

\begin{tabular}{ccc}
\hline $\begin{array}{c}\text { Serial } \\
\text { number }\end{array}$ & $\begin{array}{c}\text { PMODE } \\
{[2.0]}\end{array}$ & Introductory method \\
\hline 1 & F00 & Synchronous ADMUX (16 bit)
\end{tabular}

$\begin{array}{cc}\text { F01 } & \text { Asynchronous ADMUX (16 bit) } \\ \text { F11 } & \text { USB Boot } \\ \text { F0F } & \text { Asynchronous SRAM (16 bit) } \\ \text { F1F } & \text { IIC, if it fails, enable USB boot } \\ \text { 1FF } & \text { IIC only } \\ \text { 0F1 } & \text { SPI, if it fails, enable USB boot }\end{array}$

The firmware program in this design handles each interrupt event through a callback function. When the CYUSB3014 internal interface module generates an event, the module will trigger the corresponding interrupt, and thus the callback function corresponding to that event will be called. The communication module creates DMA channels by using the required transmit and receive sockets. If data is transferred from the device FIFO interface to the USB interface, then the P-port is used as the transmit socket and the USB as the receive socket, and vice versa. The data in this design uses the streaming input-output transfer mode for bi-directional data transfer, so two DMA channels need to be configured, one for streaming input and the other for streaming output, and the DMA channel is done by calling the DMA creation function. One is the P2U channel, that is, the GPIF II to the CYUSB3014 output channel. The PIB_SOCKET_0 is the transmit socket and the UIB_SOCKET_1 (the end connected to the upper computer) is the receive socket [17]. The throughput performance is obtained by selecting the CYUSB3014 buffer size and number. Because the two channels designed in this paper transmit a large amount of data to the host simultaneously, the overall number of buffers is fixed, so when increasing the number of buffers for one channel, the number of buffers for the other channel must be reduced. Since this design requires large data transfers to the host computer, the number of buffers for the serial input is set to 10 , and the size of each buffer is $16^{*} 1024$ bits, thus increasing the capacity of data reception. The other channel in this design is the U2P channel (from one end of the connection to the upper computer to the GPIF II interface), where PIB_SOCKET 3 is used as the receive socket and UIB_SOCKET_1 is the transmit socket. Since the commands sent by the host computer are small batch data, the number of DMA buffers for the U2P channel is set to 2, and the DMA buffer size is set to $16 \mathrm{~KB}$ for ease of operation.

With the support of the system hardware designed above, the deep neural network algorithm is used to evaluate the quality of Japanese translation and realize the system's pre-defined functions.

\section{SOFTWARE DESIGN OF JAPANESE TRANSLATION \\ QUALITY EVALUATION SYSTEM BASED ON DEEP}

\section{NEURAL NETWORK ALGORITHM}

\section{A. Japanese translation reference translation expansion}

For translations of source-end languages, the different choices of words, phrases and even word order make the existence of many correctly translated sentences, but one cannot exhaust all correct translations as reference translations to be used for translation quality evaluation. 
Using only superficial information of reference translations to evaluate systematic translations with different word order is an unfair comparison. This design represents the different translations using a compact representation like a reference translation map.

The source language sentences were chosen as the backbone and three steps were designed to create reference translation maps: first, obtaining alignment information of the source language and the reference translation; second, creating a submap of each reference translation; and finally, merging multiple submaps [18].

If $f=f_{1}, f_{2}, \ldots, f_{L}$ represents the source end of the sentence, $\quad r=r_{1}, r_{2}, \ldots, r_{n} \quad$ reference translations, translations and reference source side sentences collection consisting of $R$ reference graph is a directed graph $D(f, R)$, the directed graph is composed of point set $V$ and edge set $E$, point of each point $v$ contains two pieces of information, one is the source side fragment $\left(f_{i}, f_{j}\right)$, and the other is the translation $\left(r_{p}, r_{q}\right)$ of the corresponding fragment from the reference. In order to unify the format of the graph, a head node $\#_{s}$ and a tail node $\#_{e}$ are added to each graph, and each edge $e$ connects two corresponding nodes from adjacent source ends. For example, the node corresponding to the source segment $\left(f_{i}, f_{k}\right)$ points to the node corresponding to $\left(f_{k+1}, f_{j}\right) . P$ represents a path. The path can contain only one node or can be composed of multiple nodes, but the translation on this path must correspond to a continuous source segment. The path from the beginning point $\#_{s}$ to the end point $\#_{e}$ is a possible translation of the whole sentence at the source end. In addition, $D_{s}, D_{m}$ and $D_{t}$ are respectively used to represent the digraph formed by a single reference, the digraph formed by a monolingual corpus and the digraph corresponding to the target text.

When multiple reference translations exist, multiple subgraphs are generated, and these subgraphs need to be merged to generate the final reference translation graph. The subgraph merging starts with an empty graph $D$, and then the sets of nodes in the existing subgraphs $D_{s}$ are merged to graph $D$ one by one. First, the set of points in $D_{s}$ is merged with that of $D$. If the source end information and the target end information of two nodes are the same, the two nodes are considered to be the same node and the extra one is deleted. Then, edges are added between each node according to the process of subgraph creation [19]. For node $A\left(\left(r_{p}, r_{q}\right),(i, j)\right)$ and node $B\left(\left(r_{p^{\prime}}, r_{q^{\prime}}\right),(j+1, k)\right)$ in $D$, if there is no edge between these two nodes and there is no empty node in the neighborhood of node $B$, a new edge is added between the two nodes. After extending the reference translation graph, the translation quality evaluation index is set and the deep neural network algorithm is used to realize the evaluation of the translation quality.

\section{B. Deep neural network evaluation of translation} quality

A study on the translations obtained by several mainstream models in the current machine translation field reveals that even though these models have achieved excellent performance, they are still affected by the over-translation problem and the under-translation problem. Among them, the over-translation problem means that some words or phrases in the source language sentences are repeatedly translated in the translations, while the under-translation problem means that some words or phrases in the source language sentences are not translated in the translations.

In this paper, two new quantitative evaluation metrics, OTEM (Over-Translation Evaluation Metric) and UTEM (Under-Translation Evaluation Metric), are proposed to evaluate the processing ability of machine translation on over- and under-translation problems, respectively. Considering that usually, there can be multiple correct translations corresponding to a source language sentence, and there are differences between them in terms of word order and word choice. Besides, usually, these correct translations do not differ significantly in the information about the distribution of $\mathrm{N}$ grams in them. If the number of occurrences of an $\mathrm{N}$ - gram in a given source language sentence in the current candidate translation substantially exceeds the number of occurrences of this $\mathrm{N}$ - gram in the corresponding reference translation, it can be assumed that the current candidate translation has an over-translation problem. Similarly, if the opposite happens, it can be assumed that there is an under-translation problem in the current candidate translation.

Based on the above analysis, automatic evaluation metrics OTEM and UTEM are proposed for the over- and under-translation phenomena, respectively, based on the $\mathrm{N}$-gram matching ( $\mathrm{N}$-gram Matching) information between the candidate translation and the reference translation corresponding to the source language sentences [20]:

$$
\text { OTEM/UTEM }=L P^{*} \exp \left(\sum_{n=1}^{N} w_{n} \log m p_{n}\right)
$$

Where, $L P$ is the factor of length penalty; $N$ is the maximum length of the gram considered in the calculation of the evaluation index; $m p_{n}$ represents the proportion of $N$-grams with mismatches in the current candidate translations to be evaluated, where the mismatches include $N$-grams with no matches in the case of undertranslation and $N$-grams with multiple matches in the case of overtranslation. $w_{n}$ is weight information. The calculation formula of $m p_{n}$ is as follows:

$$
m p_{n}=\frac{\sum_{c \in\{C\}} \sum_{n-\text { gram } \in c} \text { Count }_{\text {over }}(n-\text { gram })}{\sum_{c^{\prime} \in\{C\}} \sum_{n-\text { gram }^{\prime} \in c^{\prime}} \text { Count }_{\text {cand }}(n-\text { gram })}
$$

The above formula, $\{C\}$ represents the set of candidate translations to be evaluated in the current data set. Count $_{\text {over }}$ is used to calculate the number of times of $N$-gram translation phenomena in the candidate translation. 
Count $_{\text {cand }}$ is used to calculate the occurrence number of $N$-grams in the candidate translation

When the candidate translation has been translated, the length of the candidate translation will be relatively long, because the $N$-grams in the candidate translation will be translated repeatedly, which will lead to the calculation bias of OTEM. In order to compensate for the influence of this on the calculation of evaluation indexes, the length penalty term $L P$ is introduced to punish excessively long candidate translations. The formula is defined as follows:

$$
L P=\left\{\begin{array}{l}
1, c<r \\
e^{1-\frac{r}{c}}, \text { else }
\end{array}\right.
$$

Where, $c$ represents the length of the candidate translation and $r$ represents the length of the reference translation.

The UTEM index is used to evaluate the degree of under translation of the source language information in the candidate translation. In this case, the formula of $m p_{n}$ is as follows:

$$
m p_{n}=\frac{\sum_{R \in\left\{R_{e}\right\}} \sum_{n-\text { gram } \in R} \text { Count }_{\text {under }}(n-\text { gram })}{\sum_{R^{\prime} \in\left\{R_{e}\right\}} \sum_{n-\text { gram }^{\prime} \in R^{\prime}} \text { Count }_{\text {ref }}(n-\text { gram })} \text { (4) }
$$

In the above formula, $\left\{R_{e}\right\}$ refers to the set constituted by the corresponding reference translations of candidate translations in the current data set; Count ${ }_{\text {ref }}$ is used to calculate the number of occurrences of $N$-grams in the translated text; Count under $_{\text {is }}$ the number of times that $N$-grams do not appear in the translation. At the same time, in order to avoid UDEM deviation, the length penalty term $L P$ is proposed to solve the length deviation problem. The calculation formula is as follows:

$$
L P=\left\{\begin{array}{l}
1, c>r \\
e^{1-\frac{c}{r}}, \text { else }
\end{array}\right.
$$

After establishing the above evaluation indexes, the deep neural network algorithm is used to evaluate the translation quality. The deep neural network consists of an input layer and several hidden layers. Monolayer neurons are constructed layer by layer, so that a single-layer network is trained each time. When all the layers had finished training, the tuning was performed. Change the weight between the layers except the top layer to be bidirectional, so that the top layer is still a single-layer neural network, while the other layers become a graph model. The state function of the algorithm is composed of an adder and a bias term, as shown in the following equation [21], [22]:

$$
y_{i}=\sum_{k=1}^{N} x_{k} w_{k}+b_{i}
$$

In the above formula, $x_{k}$ is the $k$ th input value in the upper layer of the network; $N$ is the number of upper-level nodes connected to this node; $w_{k}$ represents the connection strength between this node and $x_{k} ; b_{i}$ is the offset term. Parameters of deep neural network can only be established after optimization. Let the objective function of parameter optimization of deep neural network be as follows:

$$
E(W)=\frac{1}{2} \sum_{k=1}^{M}\left\|T^{k}-Z^{k}\right\|^{2}
$$

Where, $E(W)$ is the sum of the squares of the output errors of all samples; $M$ is the sample size; $T$ is the standard output value vector of the output layer; $Z$ is the actual output value vector of the output layer. The actual output value vector of the output layer can be calculated as follows:

$$
Z^{k}=f\left(Y^{k}\right)
$$

In the above formula, $Y$ is the state value vector of the output layer; $f$ is a unipolar sigmoid function. Using the training data set, the algorithm parameters are determined according to the optimization objective function of the algorithm parameters. The translation is input into the deep neural network, and the evaluation result of translation quality is output according to the calculation formula of the evaluation index. Through the above research, the deep neural network algorithm is used to evaluate the quality of Japanese translation. So far, the design of Japanese translation quality evaluation system based on deep neural network algorithm is completed.

\section{System Functional Test}

The deep neural network algorithm was used to design the Japanese translation quality evaluation system above. After completing the design of each functional module of the system, this section will conduct a functional test on the evaluation system. The evaluation system designed in this paper was compared with the neural network evaluation system based on automatic measurement, and the functional test of the system was completed by comparing the deviation between the evaluation results and the manual evaluation results as well as the data transmission and processing speed of the system.

\section{A. Test data}

1.25 million sentence pairs were extracted from the LDC database as the functional test data of the system, which contained 27.9 trillion Chinese words and 34.5 trillion Japanese words. In addition, the MTOS in the Sino-Japanese dataset is used as the development set of the algorithm training of the evaluation system, and MT02, MT03 and MT04 are used as the test set. In the test data set, each source language sentence contains the corresponding four human-translated translations as reference. In consideration of experimental efficiency, only sentences with sentence length less than 50 in the data set are retained as the training corpus of each machine translation model. In this case, $90.12 \%$ of the original data set was retained for testing.

\section{B. Test data analysis}

Figure 2 shows the deviation scatter diagram between the 
evaluation results of the two translation quality evaluation systems and the manual evaluation.

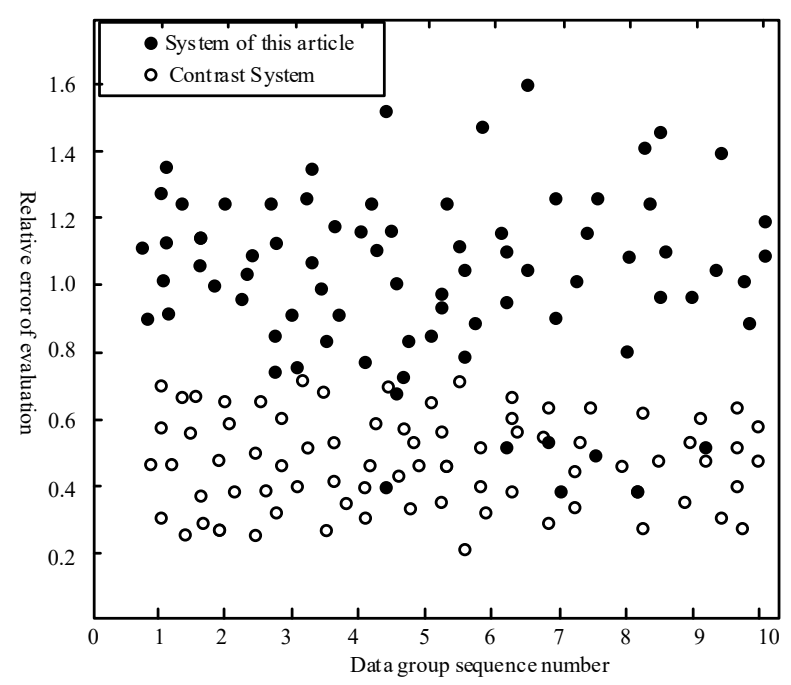

Figure 2. Test data

It can be seen from the analysis of Figure 2 that the deviation scatter distribution interval between the system designed in this paper and the manual evaluation is obviously smaller than that of the contrast system, and the numerical difference between the deviation scatter points of the system designed in this paper is relatively smaller, indicating that the evaluation result of the system in this paper is more stable. The average transmission time of the system is 5.23 seconds, whereas the manual evaluation system is 7.61 seconds, improving the processing efficiency of the design system by about $31.27 \%$. To sum up, the Japanese translation quality evaluation system based on deep neural network algorithm designed in this paper has higher processing speed and accuracy, and the evaluation results are more reliable. This is because we optimize the defects of the existing translation quality evaluation system and optimize the USB3.0 communication module of the hardware system. The reference translation of the Japanese translation was extended using the reference translation diagram. Detailed evaluation metrics were set, and deep neural networks were trained using the sample set to realize the evaluation of Japanese translation quality after determining the parameters.

\section{CONCLUSION}

As international exchanges become more frequent, relying on human translation alone can no longer meet the needs of communication, and the use of machine language translation has become the main way to improve the efficiency of text translation. However, due to the differences between different languages, machine translation has semantic bias. The quality evaluation of machine translation is an important way to promote the continuous development of machine translation technology, because only by accurately evaluating the quality of translation can we targeted improve translation technology, absorb the strengths of high-quality translation, and eliminate translation errors In view of the shortcomings of the current translation quality evaluation methods, this paper designs a Japanese translation quality evaluation system based on deep neural network algorithm. Optimize the hardware system design. The reference translation of the Japanese translation was extended using the reference translation diagram. Detailed evaluation metrics were set from both overtranslation and undertranslation, and deep neural networks were trained using the sample set to realize the evaluation of Japanese translation quality after determining the parameters. The test verified the reliability and high efficiency of the system. However, due to the limited conditions, this system is only applicable to the evaluation of Japanese translation quality, and does not have universal performance for other languages, and systems suitable for more language translation quality evaluation can be studied in the future.

\section{REFERENCES}

[1] J. Moorkens, S. Castilho, F. Gaspari and S. Doherty, "Translation quality assessment: From principles to practice", Machine Translation, vol. 33, no. 3, pp. 269-277, 2019.

[2] Z. Wang, J. R. Talburt, N. N. Wu, S. Dagtas, M. N. Zozus, "A rule-based data quality assessment system for electronic health record data", Applied Clinical Informatics, vol. 11, no. 4, pp. 622-634, 2020.

[3] L. H. Qing, "Construction of quality evaluation system for translation majors in application-oriented colleges and universities", Journal of Literature and Art Studies, vol. 9, no. 5, pp. 527-531, 2019.

[4] E. Chatzikoumi, "How to evaluate machine translation: A review of automated and human metrics", Natural Language Engineering, vol. 26, no. 2, pp. 137-161, 2020.

[5] L. Benkova, D. Munkova, U. Benko and M. Munk, "Evaluation of English-Slovak neural and statistical machine translation", Applied Sciences, vol. 11, no. 7, pp. 2948, 2021.

[6] M. A. Ali, "Quality and machine translation: An evaluation of online machine translation of English into Arabic texts", Open Journal of Modern Linguistics, vol. 10, no. 5, pp. 524-548, 2020.

[7] V. Stoykova and D. Majchrakova, "Query expansion for slovak to bulgarian language machine translation using parallel search", WSEAS Transactions on Systems and Control, vol. 16, 2021.

[8] P. Ganesh, B. S. Rawal, A. Peter and An. Giri, "POS-tagging based neural machine translation system for european languages using transformers", WSEAS Transactions on Information Science and Applications, vol. 18, pp. 26-33, 2021.

[9] S. Marzouk and S. Hansen-Schirra, "Evaluation of the impact of controlled language on neural machine translation compared to other MT architectures", Machine Translation, vol. 33, no. 1-2, pp. 179-203, 2019.

[10]L. Sandra, “The impact of respondents' multilingualism on human evaluation of machine translation quality", Jezikoslovlje, vol. 21, no. 2, pp. 207-235, 2020.

[11]I. Borch, "Lost in translation: from the university's quality assurance system to student evaluation 
practice", Nordic Journal of Studies in Educational Policy, vol. 6, no. 3, pp. 231-244, 2020.

[12] S. Muskaan, K. Ravinder and C. Inderveer, "Corpus based machine translation system with deep neural network for Sanskrit to Hindi translation", Procedia Computer Science, vol. 167, no. 1, pp. 2534-2544, 2020.

[13]M. Joss, "What to expect from neural machine translation: A practical in-class translation evaluation exercise", The Interpreter and Translator Trainer, vol. 12, no. 4, pp. 375-387, 2018.

[14]A. V. Kostin and V. V. Smirnov, "Functionality evaluation model for machine translation systems", Journal of Computer and Systems Sciences International, vol. 57, no. 1, pp. 157-169, 2018.

[15] J. Dugonik, B. Bošković, J. Brest and M. S. Maučec, "Improving statistical machine translation quality using differential evolution", Informatica, vol. 30, no. 4, pp. 629-645, 2019.

[16]B. B. Ren, "The use of machine translation algorithm based on residual and LSTM neural network in translation teaching", Plos One, vol. 15, no. 11, pp. e0240663, 2020.

[17]J. Taikyeong, "Deep neural network algorithm feedback model with behavioral intelligence and forecast accuracy", Symmetry, vol. 12, no. 9, pp. 1465-1465, 2020.

[18] X. L. Jin, Z. H. Li, H. K. Feng, Z. B. Ren and S. K. Li, "Deep neural network algorithm for estimating maize biomass based on simulated Sentinel 2A vegetation indices and leaf area index", The Crop Journal, vol. 8, no. 1, pp. 87-97, 2020.

[19]K. Stefan, S. Alexander and S. Michaela, "A deep neural network algorithm for semilinear elliptic PDEs with applications in insurance mathematics", Risks, vol. 8, no. 4, pp. 136-136, 2020.

[20] M. Afrasiabi, M. Mohammadi, M. Rastegar and A. Kargarian, "Probabilistic deep neural network price forecasting based on residential load and wind speed predictions", IET Renewable Power Generation, vol. 13, no. 11, pp. 1840-1848, 2019.

[21]C. Giuseppe and I. Gino, "Improving smart cities safety using sound events detection based on deep neural network algorithms", Informatics, vol. 7, no. 3, pp. 23-23, 2020.

[22] J. J. Q. Yu, Y. H. Hou, A. Y. S. Lam and V. O. K. Li, "Intelligent fault detection scheme for microgrids with wavelet-based deep neural networks", IEEE Transactions on Smart Grid, vol. 10, no. 2, pp. 1694-1703, 2019.

\section{Creative Commons Attribution License 4.0 (Attribution 4.0 International, CC BY 4.0)}

This article is published under the terms of the Creative Commons Attribution License 4.0

https://creativecommons.org/licenses/by/4.0/deed.en_US 